\title{
因HAD
}

DOI: http://doi.org/10.22585/hospdomic.v1i3.19

\section{Implantación de un sistema mSalud para la gestión y trazabilidad de la nutrición parenteral domiciliaria}

\author{
Implementation of an mSalud system for the management \\ and traceability of the home parenteral nutrition
}

Mercedes Cervera Peris ${ }^{1,2}$, Victor M. Alonso Roris ${ }^{3}$, Juan M. Santos Gago3, Luis M. Álvarez Sabucedo ${ }^{3}$, Carmina Wanden-Berghe ${ }^{4,5}$, Javier Sanz Valero'

1. Universidad Miguel Hernández, Elche, España.

2. Hospital Universitario Son Espases, Palma, España.

3. Escola de Enxeñaría de Telecomunicación de la Universidad de Vigo, Vigo, España.

4. Hospital General Universitario de Alicante, Alicante, España.

5. Fundación para el Fomento de la Investigación Sanitaria y Biomédica de la Comunidad Valenciana (ISABIAL FISABIO), Alicante, España.

Correspondencia/Correspondence

jsanz@umh.es

Recibido/Received

18.05.2017

Aceptado/Accepted

09.06 .2017
Conflicto de Intereses/Competing interest No existe ningún conflicto de interés en el presente estudio.

Agradecimientos/Acknowledgments

Este trabajo cuenta con una ayuda del Instituto de Salud Carlos III de Madrid, España, mediante el Proyecto de Investigación en Salud con referencia Pl13/00464 y la cofinanciación del Fondo Europeo de Desarrollo Regional - "Una manera de hacer Europa". domiciliaria. Hosp Domic. 2017;1(3): 129-39 


\section{RESUMEN}

Objetivo: Implantar un sistema mSalud para la gestión y trazabilidad de la nutrición parenteral (NP) domiciliaria.

Método: Estudio descriptivo transversal, siendo la unidad de análisis las bolsas de NP de administración domiciliaria del Hospital Universitario Son Espases de Palma (HUSE), España, durante 3 meses. Las bolsas fueron etiquetadas con un identificador unívoco (etiqueta QR) que facilitó su lectura de datos mediante un dispositivo móvil. Las etapas a controlar fueron: validación y transcripción de la prescripción, preparación, conservación y administración, siendo los puntos críticos de control el control gravimétrico, la temperatura de conservación, el volumen y tiempo de infusión (bomba de administración) y la existencia e integridad del filtro (administración). La geolocalización se realizó a través del GPS del dispositivo y la visualización mediante un Mash-up de Google Maps.

Resultados: En el proceso intervinieron 2 farmacéuticas y 20 enfermeros/as y se gestionaron 90 bolsas de NP. Las operaciones de control demostraron el cumplimiento del $100 \%$ de las etapas y del posicionamiento estático de la administración de la NP (con fecha y hora). Los resultados medios de los puntos críticos fueron: gravimetría 2217,76 $\pm 13,70 \mathrm{~g}$, temperatura de las cámaras 4,20 \pm 0,33 y 4,03 \pm 0,34, volumen a administrar 1949,81 $\pm 7,52 \mathrm{ml}$ y tiempo de infusión 15,22 \pm 0,20 h.

Conclusiones: Se ha conseguido implantar y verificar con éxito, en el HUSE de Palma (España), un sistema para la gestión y trazabilidad de la NP en la asistencia domiciliaria a un bajo coste y cuantificando los principales puntos de control.

Palabras clave: Servicios de Atención de Salud a Domicilio; Nutrición Parenteral; Aplicaciones Móviles; Control de Calidad; Validación de Programas de Computación.

\section{ABSTRACT}

Objective: To put into practice an mSalud system for the management and traceability of parenteral nutrition (PN) at home.

Methods: Descriptive cross-sectional study, taking as subject of the study the PN bags from the unit for home-care department of the University Hospital Son Espases de Palma (HUSE), Spain, during 3 months. The bags were labeled with a distinctive identifier (QR tag) that facilitated their reading of data through a mobile device. The stages to be controlled were: validation and transcription of the prescription, elaboration, conservation and administration. Critical control points to be checked are the gravimetric control, the storage temperature, the volume and infusion time (pump for administration) and the existence and integrity of the filter (administration stage). Geolocation was done through the GPS on mobile devices and it is presented using a mash-up based on Google Maps.

Results: Two pharmacists and 20 nurses took part in the process and 90 NP bags were operated. The control operations showed a $100 \%$ compliance with the established stages and the static location of PN administration (including date and time). The mean results of the critical points were: gravimetry $2217.76 \pm 13.70 \mathrm{~g}$, fridge temperature $4.20 \pm 0.33$ and $4.03 \pm 0.34$, volume to be administered $1949.81 \pm 7.52 \mathrm{ml}$ and infusion time $15.22 \pm 0.20 \mathrm{~h}$.

Conclusions: A system for the management and traceability of NP in home care at a low cost and quantifying the main control points was successfully implemented and verified at the HUSE in Palma (Spain).

Keywords: Home Care Services; Parenteral Nutrition; Mobile Applications; Quality Control; Software Validation. 


\section{INTRODUCCIÓN}

Las mezclas nutrientes parenterales, durante su proceso de preparación, conservación, distribución y administración presentan riesgos de contaminación microbiológica, química y física que hay que controlar con altos niveles de excelencia, ya que las consecuencias de estos potenciales riesgos pueden acarrear graves daños a los pacientes. Por tanto, es necesario la generación de nuevos desarrollos específicos que permitan el control de la gestión y trazabilidad de la Nutrición Parenteral (NP) y la evaluación constante (1).

La gran complejidad de esta formulación y la naturaleza interdisciplinar de la terapia nutricional hacen que sea un procedimiento especialmente vulnerable a errores (2) y, en consecuencia, es necesario establecer sistemas de evaluación/verificación que permitan asegurar la inocuidad para el paciente. Así, Martínez-Gabarrón et al (3), concluían que la gestión de la NP debe integrarse en un sistema estandarizado con el fin de asegurar la calidad y la minimización de los riesgos asociados a esta terapia, siendo el farmacéutico de hospital pieza clave en el cumplimiento de las normas de buena práctica establecidas. Igualmente, disponer de tecnologías aplicadas a la NP permitiría configurar sistemas de gestión más completos y fáciles de aplicar en un contexto real. Sin embargo, actualmente es un potencial no explotado.

La aplicación de las tecnologías de la información y la comunicación (TIC) en el ámbito de la salud abre una larga lista de posibilidades dirigidas a la consecución de grandes beneficios en multitud de niveles del entorno sanitario. Se presenta un amplio espectro de vías de investigación en este sentido, donde el uso de las TIC toma un protagonismo muy evidente en el desarrollo de los proyectos de investigación en salud y en nutrición, especialmente en entornos colaborativos que van mucho más allá de las aplicaciones Web. De esta forma, se configura un área nueva de trabajo, eSalud o eHealth, en la que surgen grandes avances, nuevos beneficios y posibilidades al aplicar las TIC al ámbito sanitario (4).

En esta línea, cabe señalar que los sistemas diseñados mediante programas informáticos permiten disponer de repositorios de los registros que relacionan y evalúan la información sobre los procesos de la NP en cualquier momento (5). Especial importancia toman las medidas para la gestión de la calidad y la trazabilidad en la NP domiciliaria (6). Además, la verificación de cualquier sistema que se desarrolle para el control de la NP permite determinar si el proceso cumple con los requisitos y condiciones impuestas en la fase de diseño de cada uno de los cometidos (7) y permite definir las tareas que se espera que el aplicativo informático llegue a completar. Debe confirmar que el sistema funciona correctamente. Las operaciones de control deben validar que se vigilan los peligros correspondientes y confirmar que el sistema funciona como estaba planeado. Así mismo, el sistema generado debe documentar los registros de los controles establecidos para demostrar que todo el proceso de la NP está bajo control y que las acciones correctivas han sido tomadas ante cualquier desviación de los puntos críticos.

Por todo lo anteriormente expresado, el objetivo de este estudio fue implantar y verificar un sistema mSalud para la gestión y trazabilidad de la nutrición parenteral domiciliaria.

\section{MÉTODO}

- Diseño: Estudio descriptivo transversal derivado de la implantación de un sistema de gestión de la nutrición parenteral domiciliaria.

- Unidad de análisis: Las bolsas de nutrición parenteral administradas en el domicilio del paciente del Hospital Universitario Son Espases (HUSE) de Palma, España. 
- Periodo del estudio: 3 meses (del 6 de junio de 2016 a 6 de septiembre de 2016).

- Sistema mSalud: sistema de eSalud en el que los usuarios utilizan dispositivos móviles para el acceso a los servicios.

- Aplicación mSalud: Utilizando una metodología basada en el análisis de peligros y aprovechando las tecnologías semánticas, se diseñó una plataforma holística de servicios para la trazabilidad y el control de procesos $(8,9)$.

- Evaluación de la plataforma: La prueba de la operatividad de la plataforma telemáticamente diseñada se recogió en el trabajo de Alonso Rorís et al (10).

- Identificación de las bolsas: Para la gestión de las etapas de validación y transcripción de la prescripción se utilizó el HospiWin $2000^{\circledR}$ versión 8, que proporcionaba la etiqueta que se adhería a cada bolsa de NP. Además, las entidades a monitorizar (las bolsas de NP) fueron etiquetadas con un identificador unívoco (fijación de una etiqueta QR).

- Variables a estudio: Las etapas a controlar se obtuvieron a partir del cuadro de gestión de cada etapa del proceso de trazabilidad de la NP (11): Validación de la prescripción, transcripción de la prescripción, preparación, conservación y administración. Las operaciones que fueron caracterizadas como Puntos Críticos de Control (PCC) fueron: Control gravimétrico, temperatura de la nevera (conservación), volumen y tiempo de infusión (administración) y existencia e integridad del filtro (administración).

- Invocación al sistema: Cada una de las solicitudes se corresponde con una orden de cumplimiento de una operación de control de una determinada etapa. En caso de que una operación fuera invocada más de una vez, por NP, el sistema solo validaba una, almacenando las restantes como «petición nula».

- Geo-localización: La aplicación móvil fue diseñada para obtener de forma automática las coordenadas de geolocalización, accesibles a través del GPS del dispositivo, en el momento del registro de las medidas de monitorización. Esto permite que cualquier registro realizado pueda ser geo-localizado de forma inmediata. Para visualizar la geo-localización de las trazas (en referencia al lugar de entrega de la NP para su administración) se usó un Mash-up de Google Maps.

- Análisis de los datos: Se llevó a cabo un estudio descriptivo de las operaciones de control asignadas para cada una de las etapas. Las variables cuantitativas se describieron con su media y desviación estándar, mediana, máximo y mínimo, y las cualitativas con su valor absoluto y porcentaje, representándose las más relevantes mediante la utilización de tablas y figuras. Para comprobar la significación en la diferencia de medias para muestras independientes se utilizó la prueba T de Student. El nivel de significación utilizado en todos los contrastes de hipótesis fue $a \leq 0,05$.

Los datos monitorizados a través del dispositivo móvil (teléfono móvil o tablet), eran enviados al servidor Web. El servidor procesaba estos datos y los registraba en la base de conocimiento en forma de hechos. El modelado de la información en esta base se diseñó utilizando las tecnologías propias de la Web Semántica. Estas tecnologías permitieron representar los conceptos y relaciones, y ofrecieron capacidades avanzadas de consulta y análisis (9).

Para el acceso a la información procesada por el sistema, el servicio Web ofrecía un cuadro de mando, un dashboard, accesible a través de un navegador Web común. En este cuadro de mando, el analista o el auditor, pudo estudiar y evaluar el histórico de registros realizados durante el uso del sistema, filtrarlos en base a diversos aspectos del contexto (la fecha, el usuario 
responsable, etc.), descargar la información en algún formato normalizado (XES o CSV) y revisar estadísticos de uso a través de gráficos fácilmente interpretables.

- Requisitos éticos: el presente trabajo forma parte del proyecto PI13/00464 y fue aprobado por el órgano evaluador de proyectos de la Universidad Miguel Hernández con el número de registro 2013.95.E.OEP.

\section{RESULTADOS}

En la implantación del sistema mSalud para la gestión y trazabilidad de la NP en enfermos domiciliarios intervinieron 2 farmacéuticas y 20 enfermeros/as y se gestionó un total de 90 bolsas de NP; ver figura 1.

Figura 1. Instante de uso del prototipo durante la elaboración de una nutrición parenteral en el servicio de farmacia del Hospital Universitario Son Espases de Palma

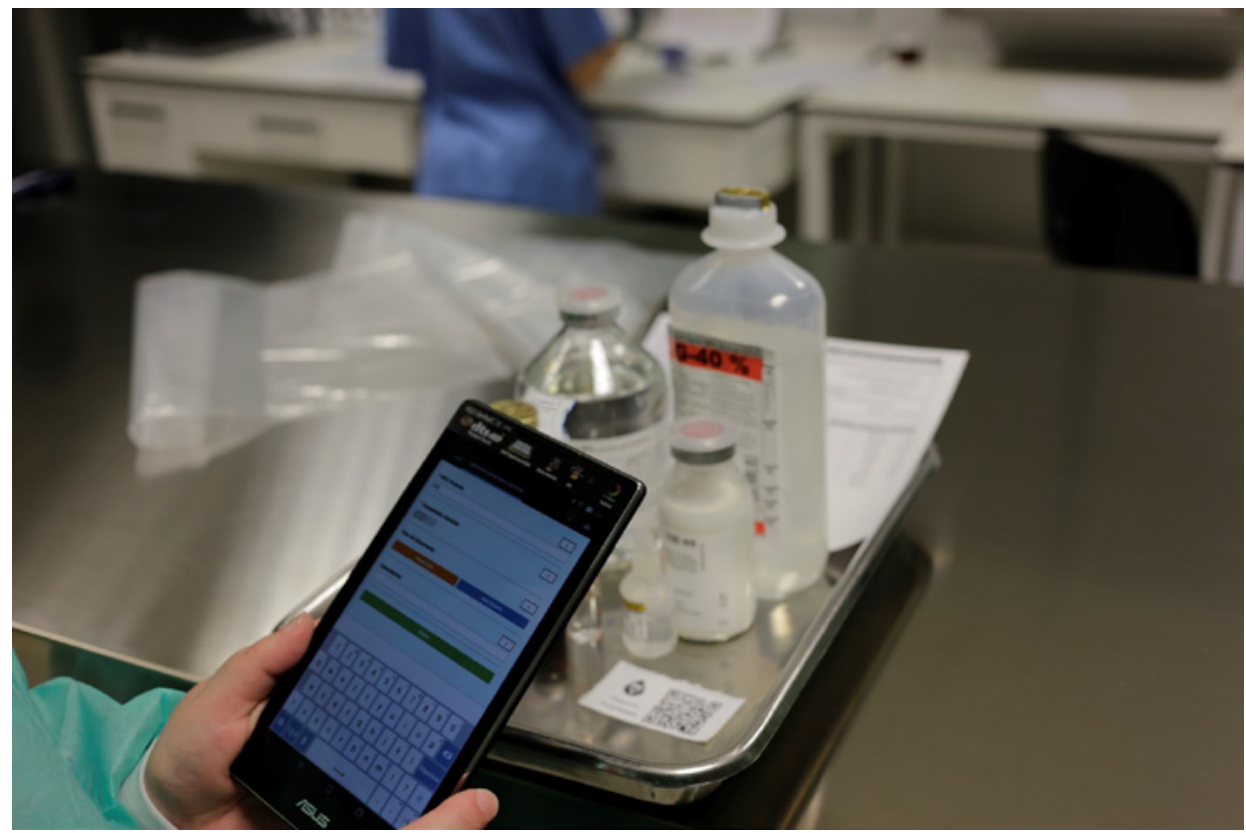

Las etapas de validación y transcripción de la prescripción, efectuadas mediante el programa informático HospiWin $2000^{\circledR}$, fueron posteriormente registradas en el sistema. Para el seguimiento global del proceso se utilizaron, adheridos a la bolsa de NP, un total de 90 códigos QR; ver figura 2 y 3. 
Figura 2. Código QR, de identificación y seguimiento, único para cada bolsa de nutrición parenteral

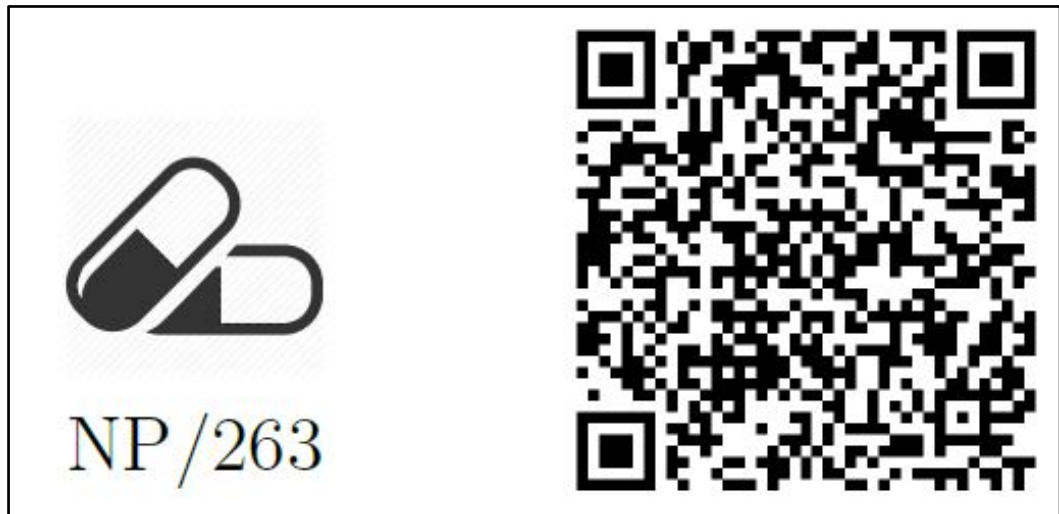

Figura 3. Bolsa de nutrición con el etiquetado y código QR para la identificación y seguimiento

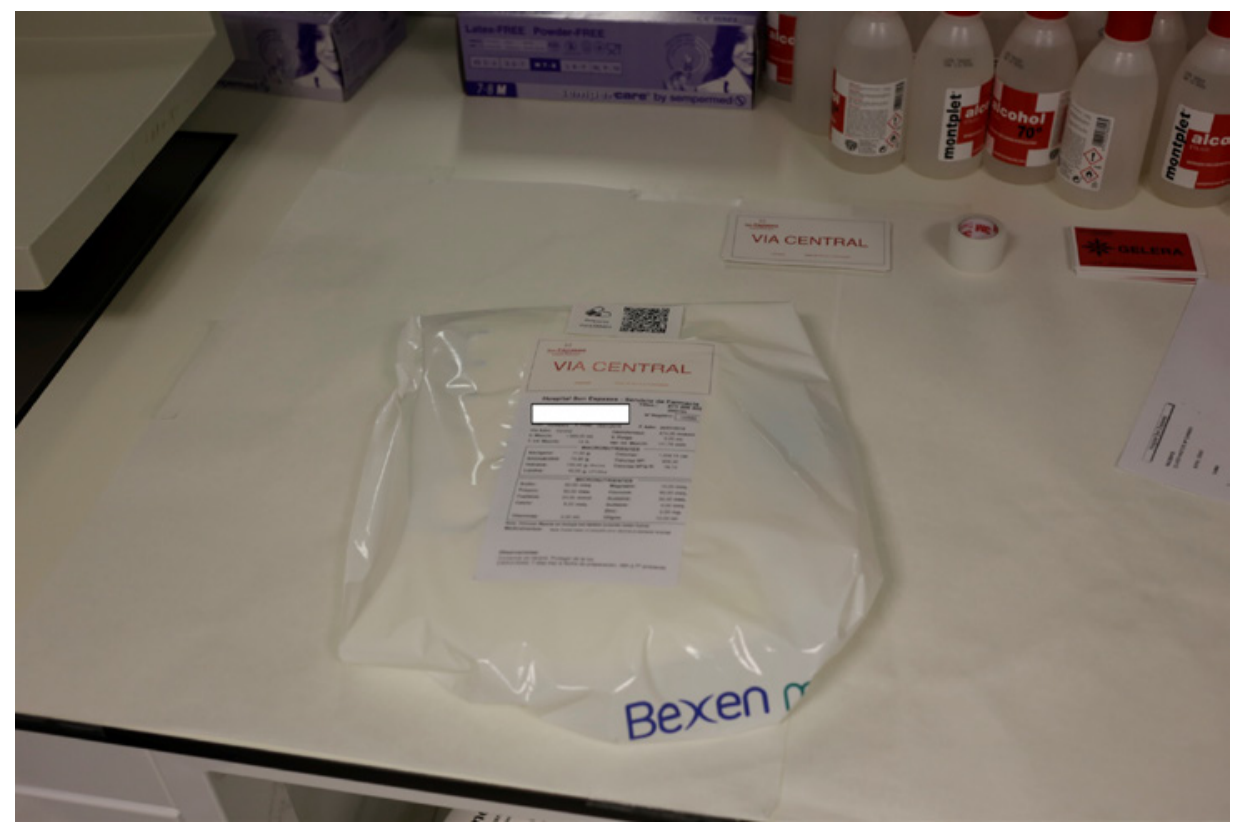

Todas las operaciones de control demostraron el cumplimiento de las diferentes etapas. En la validación y la transcripción de la prescripción se contabilizaron, en cada una de ellas, un total de 94 invocaciones al sistema (4 peticiones nulas). En la operación de control del tipo de paciente (domiciliario) perteneciente a la etapa de administración se contabilizaron 92 invocaciones. Las operaciones de control para cada etapa y las desviaciones detectadas en el conjunto de bolsas gestionadas, se pueden observar en la tabla 1. 
Tabla 1. Operaciones de control para cada etapa en el sistema de gestión y control de la trazabilidad de la nutrición parenteral (NP) domiciliaria.

\begin{tabular}{|c|c|c|c|}
\hline Etapas & $\begin{array}{l}\text { Operación de control } \\
\text { (en el sistema) }\end{array}$ & Invocaciones & $\begin{array}{r}\text { Peticiones } \\
\text { nulas }\end{array}$ \\
\hline $\begin{array}{l}\text { Validación de la } \\
\text { prescripción }\end{array}$ & Validar prescripción & 94 & $4(4,17 \%)$ \\
\hline $\begin{array}{l}\text { Transcripción de } \\
\text { la prescripción }\end{array}$ & Transcribir prescripción & 94 & $4(4,17 \%)$ \\
\hline \multirow[t]{7}{*}{ Preparación } & Comprobación inicial de bateas/materiales & 90 & -- \\
\hline & Control de la elaboración de la NP & 90 & -- \\
\hline & Comprobación final de bateas/materiales & 90 & -- \\
\hline & $\begin{array}{l}\text { Control de datos y parámetros físicos y graviméticos } \\
\text { (PCC) }\end{array}$ & 90 & -- \\
\hline & Comprobación final de la NP* & 90 & -- \\
\hline & Registro microbiológico de la NP & a posteriori** & \\
\hline & Registro microbiológico contramuestra de la NP & a posteriori** & \\
\hline Conservación & Control temperatura de la nevera (PCC) & 90 & - \\
\hline \multirow[t]{8}{*}{ Administración } & Hora de inicio & 90 & -- \\
\hline & Velocidad de infusión (PCC) & 90 & -- \\
\hline & Duración de la administración & 90 & - \\
\hline & Volumen a administrar (PCC) & 90 & -- \\
\hline & Identificación de la bomba & 90 & - \\
\hline & Existencia del filtro (PCC) & 90 & - \\
\hline & Tipo de paciente & 92 & $2(2,22 \%)$ \\
\hline & Tipo de administración & 90 & -- \\
\hline $\begin{array}{l}{ }^{*} \text { Punto de contro } \\
{ }^{* *} \text { El registro del a } \\
\text { posteriormente ur } \\
\text { PCC }=\text { Operación }\end{array}$ & $\begin{array}{l}\text { especial vigilancia. } \\
\text { isis microbiológico de la NP y, en su caso, la contram } \\
\text { ez conocidos los resultados. } \\
\text { control de una determinada etapa caracterizada con }\end{array}$ & tra se registraron & \\
\hline
\end{tabular}


Las operaciones caracterizadas como PCC, recogidas en la tabla 1, fueron controladas en las 90 bolsas de NP (100\% de los controles sobre los PCC), observándose el adecuado seguimiento. Los controles microbiológicos se realizaron según el protocolo establecido por el Servicio de Farmacia del Hospital, analizándose 1 NP por día y anotándose su conformidad a posteriori en el sistema.

Destacar el cumplimiento total (90 bolsas NP, 100\%) en el control de las bateas y sobre todo de la etapa de control final de la NP.

En la etapa de administración también hubo un 100\% de cumplimiento en la verificación del filtro de la bomba de infusión.

Los estadísticos obtenidos para el control de los PCC, de las 90 NP, pueden consultarse en la tabla 2. No se observaron diferencias significativas, en relación con la temperatura entre las dos cámaras $(p=0,729)$.

\section{Tabla 2. Estadísticos de control de las operaciones caracterizadas como Puntos Críticos de Control (PCC) en el sistema de gestión y control de la trazabilidad de la nutrición parenteral (NP) domiciliaria.}

\begin{tabular}{|l|l|r|r|r|r|}
\hline Etapas & Operación de control & Media & Mediana & Máximo & Mínimo \\
\hline \multirow{2}{*}{ Preparación } & Control gravimético (gramos) & $2217,76 \pm 13,70$ & 2135,00 & 2370,00 & 2075,00 \\
\hline \multirow{2}{*}{ Conservación } & Cámara $1-$ temperatura $\left({ }^{\circ} \mathrm{C}\right)$ & $4,20 \pm 0,33$ & 5,05 & 7,20 & 0,90 \\
\cline { 2 - 6 } & Cámara $2-$ temperatura $\left({ }^{\circ} \mathrm{C}\right)$ & $4,03 \pm 0,34$ & 5,00 & 7,20 & 0,80 \\
\hline \multirow{2}{*}{ Administración } & Tiempo de infusión (horas) & $15,22 \pm 0,20$ & 16,00 & 24,00 & 11,00 \\
\cline { 2 - 6 } & Volumen a administrar $(\mathrm{ml})$ & $1949,81 \pm 7,52$ & 1885,00 & 2028,00 & 1885,00 \\
\hline
\end{tabular}

Los resultados obtenidos en los PCC cuantitativos estaban dentro de los límites recomendados en los procedimientos normalizados de trabajo del HUSE.

Mediante la utilización de las coordenadas del GPS del dispositivo móvil se determinó el posicionamiento estático de la administración de la NP. Estos datos mostraban la posición y a la vez informaba de la fecha y hora de cada administración; ver figura 4.

Figura 4. Posicionamiento del lugar de administración de la nutrición parenteral domiciliaria con la fecha y hora en la que produjo cada una de ellas

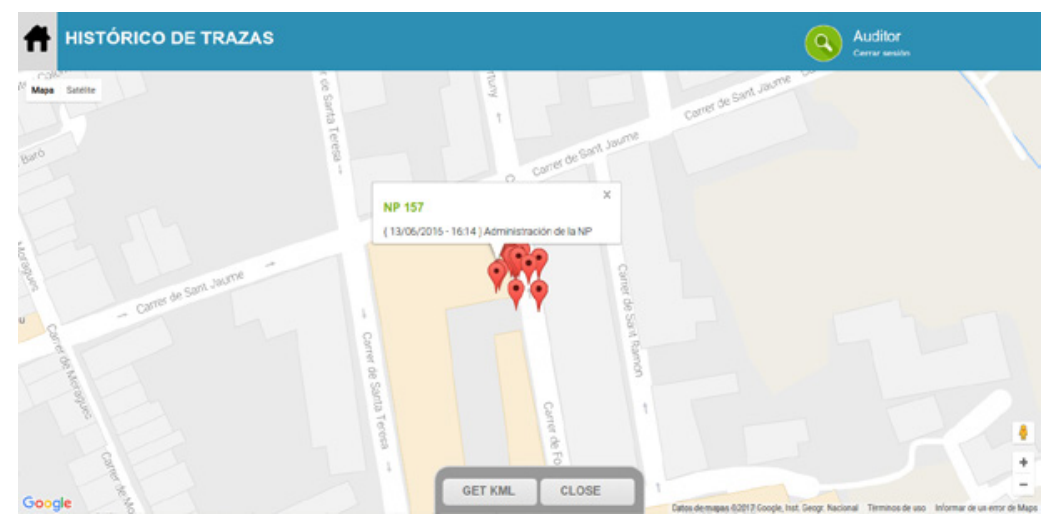




\section{DISCUSIÓN}

Este trabajo presenta cómo integrar, de forma sencilla, el control y gestión del proceso de la NP desde el servicio de farmacia hasta el paciente ingresado en el domicilio. Los puntos clave están en la identificación única e inconfundible de las bolsas de NP y la localización de la misma a lo largo de su ciclo de vida, habiendo sido implantada con éxito en el HUSE de Palma a muy bajo coste.

El cumplimiento de las diferentes etapas del proceso de la NP asegura una disminución en los potenciales riesgos asociados a la NP. La protocolización del soporte nutricional especializado disminuye drásticamente la variabilidad clínica no justificada, lo que tiene un impacto directo en la mejora de la calidad y en la minimización de los riesgos asociados con la utilización de este tipo de terapia $(12,13)$.

La caracterización y cuantificación de los PCC permitió conocer su adecuación a los resultados esperados. El control gravimétrico ayudó a garantizar en todo momento la calidad de la elaboración de la NP (14), ya que esta estrategia, de forma conjunta a otros métodos (fundamentalmente, el control de las bateas con los productos de preparación inicial y final, y la comprobación final de la NP), complementa los procesos de garantía de la NP. El histórico de datos permitió definir los «mínimos de acción» para mejorar el control de calidad de la NP. A la par, disponer de un repositorio con el control de cada etapa y de las posibles desviaciones detectadas permite perfeccionar el sistema y conocer las acciones correctoras que deben emprenderse para mejorar todo el proceso de la NP. Como indicaron Sirvent Ochando et al (15), «una vez definida la práctica de referencia con sus correspondientes grados de recomendación, resulta imprescindible disponer de una herramienta que permita cuantificar en qué medida nuestra práctica se aproxima a la establecida en el estándar (alcance o grado de cumplimiento de los estándares), y que además permita realizar el seguimiento y la trazabilidad de los resultados derivados de la implantación de acciones de mejora».

La determinación de la fecha y hora de administración, así como el volumen y tiempo de infusión que se ha llevado a cabo en esta implantación cumple con los estándares para la NP establecidos en el "modelo ASPEN" (American Society of Parenteral and Enteral Nutrition) (16). Esta sociedad científica, en sus recomendaciones (17), hace hincapié en la existencia de medidas de seguridad que eviten contratiempos relacionados con la bomba de infusión, indicando que deben contar, como mínimo con el control de velocidad y volumen exacto, controles de flujo y alarmas para detectar cambios de aire y presión en la tubería de administración. Siempre que sea posible, las bombas de infusión deben ser estandarizadas en la institución sanitaria y hay que disponer de protocolos de funcionamiento (incluyendo reglas sobre el silenciamiento de la alarma) que permitan reducir errores debidos a la programación. Evitar estos errores y capturar los datos puede apoyar programas de mejora de la calidad. A todo ello, hay que incluir la educación sobre las operaciones relacionadas con la bomba de infusión del paciente o el cuidador, especialmente en la asistencia domiciliaria (18). Todas estas recomendaciones fueron tenidas en cuenta a la hora de realizar los PCC del proceso de la NP que se propone.

Este estudio permitió controlar la aptitud de la temperatura de conservación, evitando así las alteraciones de la compatibilidad y estabilidad de las NP lo que contribuye a la seguridad del paciente, una de las dimensiones relevantes de la calidad de la atención de la salud (19).

La utilización de una interfaz de sistema de información geográfica (GIS) permitió la visualización e integración de diversas categorías de datos -localización, fecha, hora- en el control del proceso y la trazabilidad total de la NP. La viabilidad y utilidad de artefactos propios de la Web 2.0, como los Mashups, así como las tecnologías de la Web 3.0 o Web Semántica ya había sido demostrada anteriormente (20). Estas tecnologías fueron utilizadas en este trabajo para disponer de los datos de la administración de la NP y además, permitieron al equipo sanitario de Hospital a Domicilio tener la certeza y seguridad de su correcta llegada al domicilio del paciente. 
Una posible limitación a este estudio es que no existen trabajos previos sobre evaluación e implantación de estos sistemas en el control de la NP. Por ende, no es posible establecer una comparación directa ni cuantificar sus resultados. Aunque, los resultados están en línea con las actuales investigaciones sobre control de la trazabilidad de mezclas intravenosas (21) y responden a lo demandado, en una revisión sistemática (3), publicada en 2017, sobre sistemas de información en farmacia clínica aplicados a la gestión y trazabilidad de la nutrición parenteral, se manifestaba la necesidad de contar con trabajos de investigación que valorasen el establecimiento y puesta en marcha de sistemas de gestión del control y la trazabilidad de la NP.

Teniendo en cuenta todo lo anteriormente expuesto, se puede concluir que se ha conseguido implantar y verificar con éxito, en el HUSE de Palma (España), un sistema para la gestión y trazabilidad de la NP en la asistencia domiciliaria a un bajo coste y cuantificando los principales puntos de control.

\section{BIBLIOGRAFÍA}

1. Cardona Pera D, Cervera Peris M, Fernández Arévalo M, Gomis Muñoz P, Martínez Tutor M, Piñeiro Corrales G, et al; Grupo Nutrición de Farmacia de SENPE-SEFH. Consenso español sobre la preparación de mezclas nutrientes parenterales. Farm Hosp. 2009;33(Supl 1):78-107.

2. Sirvent M, Calvo MV, Pérez-Pons JC, Rodríguez-Penín I, Marti-Bonmatí E, Vázquez A, et al; Grupo de Nutrición Clínica de la SEFH. Buenas prácticas para el uso seguro de las bolsas tricamerales de nutrición parenteral. Farm Hosp. 2014;38(5):389-97. DOI: 10.7399/fh.2014.38.5.8085; PMID: 25344132

3. Martínez Gabarrón J, Sanz-Valero J, Wanden-Berghe C. Information systems in clinical pharmacy applied to parenteral nutrition management and traceability: a systematic review. Farm Hosp. 2017;41(1):89-104. DOI: 10.7399/fh.2017.41.1.10610; PMID: 28045654

4. Wanden-Berghe C. Calidad, innovación y desarrollo tecnológico en nutrición enteral en el siglo XXI. Nutr Hosp. 2015;(5):67-76. DOI: 10.3305/nh.2015.31.sup5.9133

5. Siquier Homar P, Pinteño Blanco M, Calleja Hernández MÁ, Fernández Cortés F, Martínez Sotelo J. Development of integrated support software for clinical nutrition. Farm Hosp. 2015;39(5):24068. DOI: 10.7399/fh.2015.39.5.8807; PMID: 26546938

6. Juana-Roa J, Wanden-Berghe C, Sanz-Valero J. The reality of home-based parenteral nutrition in Spain. Nutr Hosp. 2011;26(2):364-8. DOI:10.3305/nh.2011.26.2.5203; PMID: 21666975

7. Engel A. Verification, validation, and testing of engineered systems. Hoboken, N.J: Wiley \& Sons; 2010.

8. Alonso Rorís VM, Santos Gago JM, Álvarez Sabucedo L, Ramos Merino M, Sanz-Valero J. An ICT-Based Platform to Monitor Protocols in the Healthcare Environment. J Med Syst. 2016;40(10):225. DOI: 10.1007/s10916-016-0593-3; PMID: 27624494

9. Alonso-Rorís VM, Álvarez-Sabucedo L, Santos-Gago JM, Ramos-Merino M. Towards a costeffective and reusable traceability system. A semantic approach. Comput Ind. 2016;83:1-11. DOI: 10.1016/j.compind.2016.08.003

10. Alonso Rorís VM, Álvarez Sabucedo LM, Wanden-Berghe C, Santos Gago JM, Sanz-Valero J. Towards a Mobile-Based Platform for Traceability Control and Hazard Analysis in the Context of Parenteral Nutrition: Description of a Framework and a Prototype App. JMIR Res Protoc. 2016;5(2):e57. DOI: 10.2196/resprot.4907; PMID: 27269189 
11. Bernabeu Soria B, Mateo García M, Wanden-Berghe C, Cervera Peris M, Piñeiro Corrales G, Sanz-Valero J. Development of the management for parenteral nutrition traceability in a standard hospital. Farm Hosp. 2015;39(6):358-68. DOI: 10.7399/fh.2015.39.6.9689; PMID: 26618380

12. Boitano M, Bojak S, McCloskey S, McCaul DS, McDonough M. Improving the safety and effectiveness of parenteral nutrition: results of a quality improvement collaboration. Nutr Clin Pract. 2010;25(6):663-71. DOI: 10.1177/0884533610385349; PMID: 21139133

13. Dickerson RN, Hamilton LA, Brown RO. Use of a standardized progress note and assessment form for performance evaluation and quality improvement. Nutr Clin Pract. 2010;25(5):490-6. DOI: 10.1177/0884533610379607; PMID: 20962308

14. Pérez Serrano R, Vicente Sánchez MP, Arteta Jiménez M. Control gravimétrico en la nutrición parenteral: de la teoría a la práctica. Nutr Hosp. 2011;26(5):1041-4. DOI: 10.3305/ nh.2011.26.5.5125; PMID: 22072350

15. Sirvent M, Victoria Calvo M, Sagalés M, Rodríguez-Penin I, Cervera M, Piñeiro G, et al; Grupo de Nutrición de la SEFH. Indicadores de monitorización del proceso de soporte nutricional especializado. Farm Hosp. 2013;37(1):15-26. DOI: 10.7399/FH.2013.37.1.154; PMID: 23461496

16. Boullata JI, Holcombe B, Sacks G, Gervasio J, Adams SC, Christensen M, et al; Parenteral Nutrition Safety Committee (American Society for Parenteral and Enteral Nutrition). Standardized Competencies for Parenteral Nutrition Order Review and Parenteral Nutrition Preparation, Including Compounding: The ASPEN Model. Nutr Clin Pract. 2016;31(4):548-55. DOI: 10.1177/0884533616653833; PMID: 27317615

17. Ayers P, Adams S, Boullata J, Gervasio J, Holcombe B, Kraft MD, et al; American Society for Parenteral and Enteral Nutrition. ASPEN parenteral nutrition safety consensus recommendations. JPEN J Parenter Enteral Nutr. 2014;38(3):296-333. DOI: 10.1177/0148607113511992; PMID: 24280129

18. Worthington P, Balint J, Bechtold M, Bingham A, Chan L-N, Durfee S, et al. When Is Parenteral Nutrition Appropriate? JPEN J Parenter Enteral Nutr. 2017;41(3):324-77. DOI: 10.1177/0148607117695251; PMID: 28333597.

19. Tuan F, Perone V, Verdini R, Pell MB, Traverso ML. Validación de la conservación de la cadena de frío durante la distribución de mezclas de nutrición parenteral. Farm Hosp. 2015;39(5):269 74. DOI: 10.7399/fh.2015.39.5.8869; PMID: 26546939

20. Cheung K-H, Yip KY, Townsend JP, Scotch M. HCLS 2.0/3.0: health care and life sciences data mashup using Web 2.0/3.0. J Biomed Inform. 2008;41(5):694-705. DOI: 10.1016/j. jbi.2008.04.001; PMID: 18487092

21. Martínez Pérez M, Vázquez González G, Dafonte C. Safety and Traceability in Patient Healthcare through the Integration of RFID Technology for Intravenous Mixtures in the PrescriptionValidation-Elaboration-Dispensation-Administration Circuit to Day Hospital Patients. Sens Basel. 2016;16(8):E1188. DOI: 10.3390/s16081188; PMID: 27483269 